\title{
Genetically modified adoptive immunotherapy-A new therapeutic option to cure relapsed and/or refractory leukemia in children and adults?
}

\author{
Susanne Reuther* \\ Ludwig-Maximillian University Munich, Medical Faculty, University Hospital of Munich, Germany
}

\begin{abstract}
Refractory/relapsed leukemias in children, adolescents and adults are generally associated with a poor prognosis and usually require allogeneic stem cell transplantation. Despite the significant developments over the past few decades, a reliable therapy leading to long-term complete remission is still not available. Therefore, the development of adoptive immunotherapies has gained enormous importance. In recent years various leukemia-specific antibodies have been established for treatment of relapsed and/or refractory leukemias. Checkpoint inhibitors have provided significant progress in the therapy of various relapsed/refractory/metastatic tumor entities, but were not successful in highrisk leukemias. A recent milestone in the development of new specific treatment for relapsed/ refractory leukemias are genetically engineered $T$ cells expressing a Chimeric Antigen Receptor that targets specific antigens (CAR-T cells). These CAR-T cells represent a combination of gene and immunotherapy with impressive results in the treatment of pediatric and young adult patients with aggressive acute lymphoblastic leukemia or diffuse large B-cell lymphoma and in the treatment of adults with primary mediastinal B-cell lymphoma. This review summarizes the current possibilities of adoptive immunotherapies, with a focus on leukemias and the situation in Germany.
\end{abstract}

Keywords: Relapsed/refractory leukemia, Cancer, Adoptive immunotherapy, Monoclonal antibodies; CAR-T cell therapy

Accepted on 21 December 2018

\section{Introduction}

\section{Leukemia in children and adults}

The term leukemia comprises several types of cancer of the blood cells, which are mainly classified according to the type of blood cells that are affected by the tumor (lymphoid or myeloid) and the rate of progression (acute or chronic) [1,2]. Leukemia occurs particularly in elderly and children.

In Germany up to 12,000 people suffer from leukemia every year including about 600 children and adolescents [3]. Leukemia accounts for about $32 \%$ of all cancers in children under the age of 15 years [4]. The most common diagnosis in children is Acute Lymphoid Leukemia (ALL, $75 \%$ of all leukemias), which is most frequent among two- to four-yearold children as compared to other age groups [4]. About $4 \%$ of all childhood malignancies and $13 \%$ of all leukemias are cases of Acute Myeloid Leukemia (AML), which is the most common type of leukemia among children under two years of age (Figure 1A) [4]. In both types of leukemia, boys are affected more frequently than girls $[4,5]$. The probability of long-term survival AML (71\%) is lower than with ALL (90\%) [4].

About $8 \%$ of patients develop secondary neoplasms within 30 years after diagnosis of leukemia during childhood and about $11 \%$ of secondary neoplasms developing within 30 years after primary cancer during childhood after are AML (Figure 1B) [4]. Malignant neoplasms are the second leading cause of death in children [6]. Furthermore, $23 \%$ of all secondary neoplasms are Central Nervous System (CNS) tumors, followed by skin tumors, thyroid cancer and breast cancer in young women [6].

While ALL is the most prevalent form of leukemia among children and adolescents, older people are more likely diagnosed with chronic forms like Chronic Lymphoblastic Leukemia (CLL) and Myelodysplastic Syndrome (MDS) [4]. More than $50 \%$ of all leukemias occur in the age group over 65 [5]. The overall incidence of this disease is higher in men than women. About one in 56 men (1.78\%) and one in 79 women $(1.26 \%)$ are affected by leukemia during their lifetime [5]. The reason might be a gender-specific genetic difference near to the ABO gene locus on chromosome 9 [7].

\section{Prognosis of patients with leukemias}

The prognosis of children and adolescents with both AML and ALL has improved significantly in the last three decades $[5,8]$.

In 2010 the five-year survival rate for children with ALL was $30 \%$ higher than it had been in 1975 , and for adolescents it was improved by more than $45 \%$ [9].

According to the Childhood Cancer Registry, today's cure rate is about $80 \%$ for childhood ALL and 30 to $70 \%$ for childhood AML $[8,10-12]$. The five-year survival rate for adults reached $84 \%$ for CLL and $69 \%$ for CML in 2014 [5]. The main reasons for the improved prognosis are the establishment of more specific diagnostics, a modern combination of therapies, prevention of the spread to the Central Nervous System (CNS) 
and risk-adapted therapy depending on prognostic factors $[12,13]$.

\section{Initial treatment options for leukemia}

Overall, with respect to AML and ALL, a long-term survival rate of up to $65 \%$ and $80 \%$, respectively, has been achieved with the aid of intensive chemotherapies [13,14]. Acute leukemias are initially treated with chemotherapy, possibly supplemented with CNS irradiation, aiming to completely eliminate the leukemic clone so that residual leukemic cells can no longer be detected, and a normal hematopoiesis can start again.

A Types of leukemias, myeloproliferative and myelodysplastic diseases in children under 15 years of age
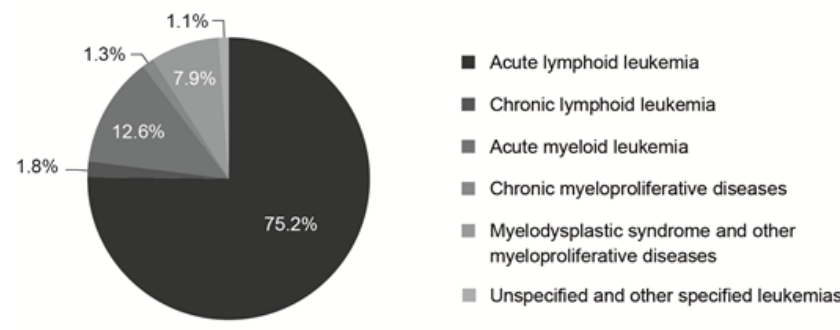

B Leukemias as secondary neoplasms within 30 years after diagnosis of any primary cancer during childhood
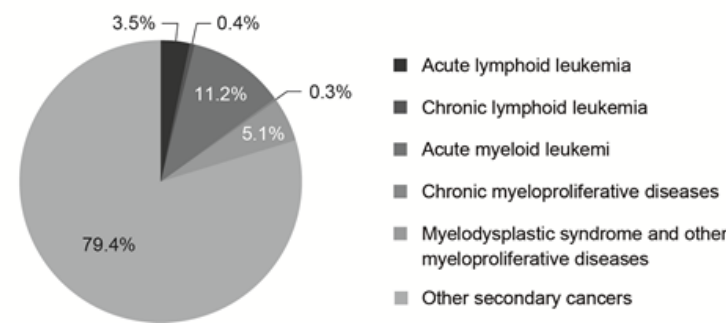

Figure 1. Leukemia diagnoses in childhood and as secondary neoplasms in Germany [4]. (A) Types of leukemias, myeloproliferative and myelodysplastic diseases in children under 15 years of age. (B) Leukemias as secondary neoplasms within 30 years after diagnosis of any primary cancer during childhood.
However, with respect to ALL, the therapy still fails in over $50 \%$ of infants [15] regarding AML, about $30-40 \%$ of pediatric patients suffer a relapse associated with poor prognosis [16]. Viral and bacterial infections contribute to mortality. If chemotherapy is not sufficiently successful, Bone Marrow Transplantation (BMT) or hematopoietic Stem Cell Transplantation (SCT) are the only curative therapeutic option in these types of leukemia as well as numerous other hematologic, oncologic and immunologic diseases [17-20]. Despite the significant developments over the past two to three decades, Graft-versus-Host Disease (GvHD) after stem cell transplantation is still the major complication, at different frequencies and degrees of severity depending on the Human Leukocyte Antigen (HLA) compatibility between donor and recipient [21].

Until today, no reliable therapy leading to long-term complete remission is available, neither for adult nor pediatric high-risk leukemia patients. Therefore, the development of an adoptive immunotherapy without inducing GvHD or other lifethreatening complications, especially in the context of haploidentical transplantation, has gained enormous importance. This review elaborates on the promising adoptive immunotherapies that have been developed in the last years, their success in the treatment of leukemias and remaining issues especially regarding refractory/relapsed leukemias, with a focus on the therapeutic landscape in Germany.

\section{Immunotherapies for treatment of leukemias- overview}

Leukemic cells often develop mechanisms to modulate the immune response. Immunotherapies comprise treatments that enhance or repress the patient's immune system to fight the cancer. These therapies can be nonspecific or antigen-specific and employ different mechanisms to manipulate the immune system. In addition, a differentiation can be made between cellular and non-cellular therapy (Table 1). The latter comprises antibodies against tumor cells, immune checkpoint inhibitors and immunomodulators like cytokines.

Table 1. Types of immunotherapies for treatment of leukemia. Immunotherapies can be differentiated between cellular and non-cellular and according to their specificity for antigens

\begin{tabular}{|l|l|l|}
\hline \multirow{2}{*}{ Immunotherapy } & Antigen-specific & Nonspecific \\
\hline \multirow{2}{*}{ Cellular } & Adoptive cell therapy & Donor Lymphocyte Infusion (DLI) \\
\cline { 2 - 3 } & Genetically modified T cells (CAR-T cells) & NK cells \\
\cline { 2 - 3 } Non-cellular & Tumor-specific antibodies & Cytokines \\
\cline { 2 - 3 } & Immune checkpoint inhibitors & \\
\end{tabular}

In adoptive immunotherapy, cells are transferred into the patient. A mixture of immune cells is transferred in Donor Lymphocyte Infusion (DLI). Single types of immune cells such as $\mathrm{T}$ and $\mathrm{B}$ lymphocytes or antigen presenting cells, that are activated specifically or nonspecifically, can be used as well.
Another classification can be made based on whether the therapy activates the patient's own immune system (active) or whether the patient is not able to produce the immune molecules on his own (passive).

It is possible to combine several types of immunotherapy to achieve a better therapeutic result. Next, nonspecific 
Citation: Susanne Reuther. Genetically modified adoptive immunotherapy-A new therapeutic option to cure relapsed and/or refractory leukemia in children and adults? J Med Oncl Ther 2018;3(2):36-45.

immunotherapies will be described in further detail followed by antigen-specific therapies.

\section{Donor lymphocyte infusions are used to treat and prevent relapse after stem cell transplantation}

To improve the therapeutic potential of allogeneic SCT, patients can receive a DLI, an infusion of lymphocytes from the stem cell donor, in order to maximize the Graft-versusLeukemia $(\mathrm{GvL})$ effect (cytotoxic effect of T-lymphocytes from the donor graft against remaining leukemic cells in the recipient). The use of DLI, which was established by Kolb et al. in 1990, has already resulted in a major therapeutic breakthrough in adult patients with CML [22]. It has been demonstrated that DLI can induce long-term complete remission in a lot of CML patients [22-24]. With regard to AML and ALL, DLI showed significantly lower therapeutic success. The reasons are both a lower or altered expression of the AML and ALL antigens [25-28] and the loss of the HLA complex, a so-called immune escape mechanism [29,30]. DLI also failed in the treatment of pediatric high-risk leukemias, or the positive effects could only be observed temporarily [31]. In addition, DLI is still limited by the risk of GvHD, especially at high T-cell doses $[32,33]$. To optimize the therapeutic potential of allogeneic SCT and minimize the graft-versus-host effect, immunotherapeutic approaches besides DLI are necessary.

In order to regulate or prevent an unwanted GvHD or immune escape and further tumor progression, a targeted modulation of the immune system is key to stimulating its own unique and highly effective mechanisms.

\section{Natural killer cells may protect against GvHD}

An approach to circumvent the problems associated with DLI constitutes the use of Natural Killer (NK) cells. As part of the innate immune system, NK cells have been shown to specifically eliminate leukemic cells after allogenic haploidentical SCT without inducing GvHD although the underlying mechanisms remain to be elucidated [34,35]. Restoration, modification and combination of NK cells with other treatments are new strategies for cancer immunotherapy [36].

\section{Immunomodulators can enhance the anti-tumor activity of the immune system}

Cytokines are important mediators of cell signaling and play a key role in the regulation of the immune system. Interferon alfa-2a has been approved for treatment of patients with hairy cell leukemia and Philadelphia chromosome positive $(\mathrm{Ph}+)$ CML while interferon alfa-2b can be applied to patients with hairy cell leukemia and aggressive follicular non-Hodgkin lymphoma [37,38]. Interferons have been shown to increase the efficacy of other treatments when used in combination, for instance in case of the tyrosine kinase inhibitor imatinib for the treatment of CML $[38,39]$. Since interferons apparently do not cross the placental barrier, they may even offer the possibility to treat leukemia in pregnant women [40]. All interferons are produced naturally by the body, the treatment can still cause side effects ranging from flu-like symptoms to reduced leukocyte and platelet counts as well as psychological effects [41]. However, side effects typically diminish after withdrawal. The side effects of interferons also impede the combination with tyrosine kinase inhibitors like imatinib [39]. Due to their dose-limiting toxic effects and inconsistent efficacy as monotherapy, future applications may be engineered cytokines or combination therapies [42].

\section{Targeted therapies reduce side effects but induce escape mechanisms}

While the standard chemotherapy targets all types of fast dividing cells, tumor cells and normal body cells, targeted therapy specifically aims at tumor cells. Underlying mechanisms include the prevention of cell growth by blocking growth promoting signals, the induction of tumor cell death, e.g. by delivering toxic compounds to the tumor cell, and the triggering of the immune system (immunotherapy) [43]. The efficacy of the immunotherapies against leukemia is determined by the complex interaction of numerous players of the immune system with the tumor cell [35]. Targeted therapies are advantageous because they may cause fewer side effects but they also come with limitations. Tumor cells can escape the targeted therapy by mutation of the target or by activation of additional pathways that are not targeted. Drugs directed against new targets are being developed but are by no means available for each target [43]. Targeted therapies include monoclonal antibodies, tyrosine kinase inhibitors, checkpoint inhibitors and CAR-T cell therapy

\section{Monoclonal antibodies are a passive, tumor-specific immunotherapy}

Passive immunotherapies such as monoclonal tumor-specific antibodies for the treatment of B-cell lymphomas and for subsets of patients with CLL, also as a first-line therapy, have been established since the approval of rituximab, a monoclonal antibody that targets the CD20 pathway, in 1997 [44]. Another 13 tumor-specific monoclonal antibodies have been approved since then, are used successfully [45] and have given a new hope to cure refractory/high-risk leukemia in children and adults. One example is Inotuzumab ozogamicin, an antibody targeting CD22 conjugated with the toxin calicheamicin. Inotuzumab ozogamicin is approved by the European Medicines Agency (EMA) for use in patients with relapsed or refractory CD22-positive B-cell ALL. In patients with $\mathrm{Ph}+$ relapsed or refractory CD22-positive B-cell ALL, treatment with at least one tyrosine kinase inhibitor should have failed before Inotuzumab ozogamicin is considered for treatment [46].

\section{Tyrosine kinase inhibitors are cytostatic but not cytotoxic}

Tumor cells are characterized by abnormal growth and proliferation. Tyrosine Kinase Inhibitors (TKIs) suppress the growth signals that tumor cells receive by blocking the signal transduction that is mediated by tyrosine kinases. 


\section{Imatinib mesylate}

Imatinib mesylate, a selective inhibitor of the BCR-ABL kinase, is approved for the treatment of newly diagnosed $\mathrm{Ph}+$ ALL in children and adults in combination with chemotherapy and for adults with relapsed or refractory $\mathrm{Ph}+$ ALL as monotherapy. Further areas of application are chronic eosinophilic leukemia or hypereosinophilic syndrome as well as $\mathrm{Ph}+\mathrm{CML}$ and further types of cancer $[47,48]$. Further therapeutic options targeting the BCR-ABL kinase, especially for patients who are refractory or intolerant to imatinib mesylate, are dasatinib, nilotinib, bosutinib, ponatinib and idelalisib.

\section{Dasatinib}

Dasatinib is a treatment option considered for adult patients with Chronic Phase (CP), Accelerated Phase (AP) or Blast Phase (BP) CML with resistance or intolerance to prior therapy including imatinib mesylate, for adults with $\mathrm{Ph}+\mathrm{ALL}$ and lymphoid blast CML with resistance or intolerance to prior therapy and for children and adults who were newly diagnosed with CP Ph+ CML [49]. Furthermore, dasatinib is indicated for children with $\mathrm{Ph}+\mathrm{CML}$ resistant or intolerant to prior therapy including imatinib mesylate [50,51].

\section{Nilotinib}

Nilotinib is also indicated for $\mathrm{CP} \mathrm{Ph}+\mathrm{CML}$ in children or adults who are newly diagnosed, and for $\mathrm{Ph}+\mathrm{CML}$ in children (CP) or adults (CP or AP) with resistance or intolerance to prior therapy including imatinib mesylate [49,51].

\section{Bosutinib}

Another treatment option for newly diagnosed adult $\mathrm{CP} \mathrm{Ph}+$ CML patients is Bosutinib, which is furthermore used in adults with $\mathrm{CP}, \mathrm{AP}$, or $\mathrm{BP} \mathrm{Ph}+\mathrm{CML}$ who were already treated with at least one tyrosine kinase inhibitor and for whom imatinib, dasatinib and nilotinib are no treatment options [52].

\section{Ponatinib}

Ponatinib is approved for adults with CP, AP or BP CML who are intolerant to dasatinib or nilotinib, for whom imatinib is not appropriate, or who carry a T315I mutation. Ponatinib can also be used for adults with $\mathrm{Ph}+\mathrm{ALL}$ who are resistant to dasatinib, intolerant to dasatinib and imatinib not applicable, or who carry a T315I mutation [53].

\section{Idelalisib}

Idelalisib can be used for treatment of CLL after at least one prior therapy or in adults with $17 \mathrm{p}$ deletion or TP53 mutation and no other therapy option [54]. Idelalisib is furthermore a treatment option for Follicular Lymphoma (FL) refractory to two prior lines of treatment, Marginal Zone Lymphoma (MZL), Small Lymphocytic Lymphoma (SLL), and, Waldenström Macroglobulinemia (WM) or lymphoplasmacytic lymphoma $[54,55]$.

Although TKIs stop the proliferation of tumor cells, they are not cytotoxic. Therefore, patients need continuous treatment to keep the cancer in check. Unfortunately, tumor cells can develop resistance against targeted therapies like imatinib. To counteract resistance development, combined therapies are applied. TKIs are the treatment of choice for CML in the chronic phase to continuously control the minimal residual disease but, as other targeted therapies, they come with high therapy costs as they are highly selective and used in small subgroups of patients.

\section{Leukemia-specific conjugated antibodies}

Monoclonal antibodies can perform their action either by binding alone or by specifically killing leukemic cells if conjugated with cytotoxic molecules. Currently 10 approved immunotherapy options as targeted antibodies for leukemia are established, which comprise conjugated antibodies and checkpoint inhibitors, which are elaborated in the next section.

\begin{abstract}
Alemtuzumab
Alemtuzumab is a monoclonal unconjugated antibody that targets the CD52 pathway via the activation of antibodydependent cell-mediated cytotoxicity [56]. It is used in adult patients who have been treated with alkylating agents and in whom the fludarabine therapy has failed. In addition, improvement of GvHD could be shown in a study where alemtuzumab was used off-label in 20 patients with severe steroid-resistant acute intestinal GvHD after allogeneic SCT yet at the cost of complications such as the reactivation of cytomegalovirus, bacterial infections and invasive aspergillosis [57].
\end{abstract}

\section{Gemtuzumab ozogamicin}

Gemtuzumab ozogamicin is a monoclonal antibody-drug conjugate consisting of a humanized monoclonal antibody targeting the CD33 pathway, conjugated with a bacterial toxin that delivers toxicity to cancer cells. Gemtuzumab ozogamicin is used to treat subsets of adult and pediatric patients over 15 years of age with newly diagnosed primarily refractory CD33positive AML, excluding Acute Promyelocytic Leukemia (APL), as a combined first-line therapy [58,59]. It was the first approval for such an antibody-toxin conjugate worldwide and the only AML therapy targeting the CD33 antigen, which is expressed on AML cells in up to $90 \%$ of patients [58]. Disadvantages are side effects that can vary between mild and very severe (e.g. neutropenia and/or thrombocytopenia, liver toxicity and sinusoidal obstruction syndrome) and furthermore the antibody is only used for a small patient group.

\section{Inotuzumab ozogamicin}

Inotuzumab ozogamicin is an antibody-drug conjugate used as a monotherapy which targets the CD22 pathway and is cytotoxic to cancer cells. It is only effective in a small group of adult patients with relapsed/refractory acute CD22-positive precursor B-ALL. A limiting factor is the increased occurrence of hepatic sinusoidal obstruction as an overlapping complication of allogeneic SCT after the application of the antibody [60,61]. 
Citation: Susanne Reuther. Genetically modified adoptive immunotherapy-A new therapeutic option to cure relapsed and/or refractory leukemia in children and adults? J Med Oncl Ther 2018;3(2):36-45.

\section{Obinutuzumab}

Obinutuzumab is a monoclonal antibody that targets the CD20 pathway to treat subsets of adult patients with CD20-positive refractory CLL and/or FL, the most common type of indolent (slow-growing) non-Hodgkin's lymphoma as first-line therapy [62]. It acts as a CD20 antibody similar to rituximab and ofatumumab, but is the first generation CD20 monoclonal antibody optimized with glycoengineering technology (glycosylation). Destruction of malignant B lymphocytes can be improved by activating other immune cells against cancer cells or directly triggering cell death. A German clinical trial showed that obinutuzumab achieved better results in treating comorbid patients with CLL compared to rituximab. On average, progression-free survival was extended to 10 months, thus replacing rituximab and chlorambucil with obinutuzumab in the standard therapy. However, obinutuzumab caused infusion reactions and neutropenia more frequently [63].

\section{Ofatumumab}

Ofatumumab is a monoclonal antibody that targets CD20positive $B$ cells. It is used to treat subsets of adult patients with refractory CD20-positive B-CLL who did not respond adequately to treatment with fludarabine or alemtuzumab or who are resistant to rituximab [64,65]. Following the onset of fulminant hepatitis upon therapy with ofatumumab, a "redhand letter" (a German pharmaceutical warning letter) was issued on December 2013. Therefore, hepatitis B virus screening is recommended before starting treatment [66].

\section{Rituximab}

Rituximab is a chimeric monoclonal anti-CD20 antibody that targets CD20 and is used as a drug in cancer immunotherapy mainly in the treatment of malignant lymphoma. The variable part of the antibody is directed against the cell surface molecule CD20, which can be detected in a part of nonHodgkin's lymphoma patients and indicated in combination with chemotherapy for treatment of previously untreated patients and for subsets of adult patients with relapsed/ refractory B-CLL, also as a first-line therapy [67-69]. Rituximab has also been found to reduce the need for splenectomy in patients with stage I splenic diffuse large B-cell lymphoma [70]. Furthermore, rituximab is the most important drug in the treatment of transplantation-associated lymphoma (PTLD), which is often CD20-positive [71].

Side effects of treatment with rituximab are neutropenia and, due to the weakened immune defense, more severe infections. In a study investigating rituximab as maintenance treatment in elderly patients, $53 \%$ of the treated patients and only $36 \%$ of the untreated group suffered from neutropenia [72].

\section{Blinatumomab}

Another small therapeutic breakthrough has been achieved with blinatumomab, a monoclonal Bispecific T-cell Engager (BiTE) antibody directed simultaneously against the CD3 receptor of $T$ cells and against the surface protein CD19 of $B$ cells. It is being used prior to allogeneic BMT in adult patients with specific forms of refractory/relapsed pre-B ALL. Blinatumomab is the first therapeutically used BiTE antibody $[73,74]$.

\section{Checkpoint inhibitors activate the immune system against tumor cells}

A new central point of immuno-oncology are checkpoints, control sites of the immune system located on T-cells. They normally prevent an over-stimulating immune reaction and thus autoimmune diseases. However, cancer cells can use this mechanism to camouflage themselves. In the last years, antibodies have been developed to attack these checkpoints [75].

CTLA-4 (cytotoxic T-lymphocyte antigen 4), ligands CD80 and CD86, PD-1 (programmed death 1), PD-L1 and PD-L2 are currently used therapeutically as target structures. This leads to activation of $\mathrm{T}$ cells, proliferation and infiltration of lymphocytes into the tumor, thus death of tumor cells [76].

\section{Ipilimumab}

In 2011 Ipilimumab became the first approved antibody which targets the $\mathrm{T}$ cells and not the tumor cell. This specific antibody relieves the cancerous blockade by attenuation of the inhibitory signal of CTLA-4, thus acting as an enhancer of T cell function [77]. A phase III clinical trial in 2011 showed a significant increase upon combined use of ipilimumab and the cytostatic agent dacarbazine in overall survival compared to chemotherapy alone [78]. In this setting, ipilimumab prolonged the survival of patients with metastatic melanoma by 2-4 months [79]. Thus, the non-specific activation of $\mathrm{T}$ cells can lead to the regression of tumors and immune cells. Thus, CTLA-4 can be considered as target structure for tumor therapy.

\section{Pembrolizumab}

The first monoclonal anti-PD-1 antibody (PD-1 inhibitor), pembrolizumab, was approved in September 2014 by the US Food and Drug Administration or the treatment of advanced melanoma following previous ipilimumab and serine/ threonine-protein kinase B-Raf (BRAF) inhibitor therapy. It reduces checkpoint-mediated blockade of tumor-reactive $T$ cells, thus restores their ability to specifically target tumor cells [80].

\section{Nivolumab}

Another monoclonal checkpoint inhibitor, nivolumab, has been available since 2015 as a PD-1 blocker and is used to treat nonresectable or metastatic melanoma and locally advanced or metastatic Non-Small Cell Lung Carcinoma (NSCLC) as well as other entities of cancer [81].

These new antibodies offer a perspective for patients for whom only very limited therapeutic options were available so far, and survival rates of such patients have improved significantly with these antibody therapies. However, their effect usually sets in only after a few weeks, and not everyone responds to immune 
checkpoint inhibitors. So far, it is unclear which patients respond to treatment and why treatment fails in others. There are no reliable predictive factors that could be used to determine the expected response before starting therapy. In addition, new immunotherapies have side effects including all overshooting immune reactions (e.g. fever or cytokine release syndrome) that can vary from mild to severe and even lifethreatening. Therefore, it is important to weigh the benefit and risk of such therapy in order to provide the patient with a good quality of life.

While checkpoint inhibitors have revolutionized therapy of melanoma and many solid tumors, the efficacy of these inhibitors is still being explored in leukemias such as AML [82].

\section{The CAR-T cell therapy gives new hope for relapsed and refractory leukemias}

Currently, great hopes rest on the new CAR-T cell therapy, a combination of gene- and immunotherapy, showing impressive success rates. CAR-T cell therapies work with the patient's own immune cells, leukocytes filtered from patient's blood via leukapheresis and modified in the laboratory to detect and kill tumor cells. These genetically engineered $\mathrm{T}$ cells, which express a chimeric antigen receptor, provide a promising treatment option especially for children and adults with relapsed and refractory B cell leukemias. The field of CAR-T cell therapy is now booming with over 240 CAR-T clinical trials running. In July 2018, two CAR-T cell therapies were approved in the EU for treatment of pediatric and adult patients with aggressive acute lymphoblastic leukemia, Diffuse Large B-Cell Lymphoma (DLBCL) (tisagenlecleucel, axicabtagene ciloleucel) or Primary Mediastinal B-Cell Lymphoma (PMBCL) (axicabtagene ciloleucel) [83,84]. These are the first CAR-T cell therapies that were approved, more are likely to follow.

\section{Tisagenlecleucel, axicabtagene ciloleucel}

Tisagenlecleucel and axicabtagene ciloleucel are used for treatment of pediatric and young adult patients up to the age of 25 years with B cell ALL who are refractory, who have relapsed after SCT, or have a second or later relapse and adult patients with relapsed or refractory Diffuse Large B-Cell Lymphoma (DLBCL) following two or more systemic lines of therapy [85]. In clinical trials, tisagenlecleucel (targeting the surface marker CD19 on malignant lymphoma cells) achieved response rates of nearly $90 \%$ in young ALL patients. Forty percent of patients with large B-cell lymphoma were able to suppress the cancer with axicabtagene ciloleucel treatment. The drug also helped patients with ALL and CLL [86].

Recent developments concerning other surface markers (CD22) could also be used in cases where CAR-T immunotherapy against CD19 does not work due to antigen loss. First clinical trials have shown promising results for patients with relapsed or refractory pre-B cell ALL [84,87-89]. These results have met the needs and expectations of patients, physicians, scientists and investors, but it is important to remember that patients may not always respond to the therapy.
Since this method can trigger very serious side effects, treatment requires special precautions and preparations.

\section{Problems associated with the CAR-T cell therapy}

Tisagenlecleucel is an autologous immunocellular cancer therapy that reprograms the patient's own $\mathrm{T}$ cells with a transgene encoding a Chimeric Antigen Receptor (CAR) to identify and eliminate CD19-expressing B cells. The extracellular portion of CAR consists of a mouse antibody that recognizes the antigen CD19 of leukemia cells. The intracellular portion of the CAR is configured to trigger a strong immune response, which comprises the proliferation of the genetically modified CAR-T cells and the release of cytokines to recruit other immune cells to attack the leukemia cells [84]. Although there are no other cells attacked, because only B cells carry CD19, there is the risk that even healthy B cells will be attacked by CAR-T cell therapies, leading to weakening of the immune system. Therefore, B-cell deficiency with concomitant immunodeficiency is an expected side effect of these therapies. However, this can be countered by a longterm treatment with antibody preparations [90].

As cancer cells differ from normal body cells in only a few features, it is almost impossible for researchers to construct the perfect artificial CAR. Hence, the effect of CAR-T cells is difficult to control and the risk of damaging the normal tissue remains, resulting in toxicities and side effects, which can become life-threatening-or lethal [91], including cytokine release syndrome. In the international ALL study with the CAR-T agent tisagenlecleucel, $77 \%$ of patients on average had a cytokine storm after 3 days, about half of whom had to be treated in the intensive care unit. Neurological symptoms (CAR-T encephalopathy syndrome, CRES) occurred in $40 \%$ of patients, but mostly disappeared in the course of treatment [86].

The careful selection of patients helps to avoid side effects or to keep them minimal. When utilizing this therapy, the immune system must not be inhibited, e.g. to avoid GvHD, there must not be uncontrolled infections. Active GvHD is also an exclusion criterion for the CAR-T immunotherapy, and the combination with DLI after SCT is contraindicated [92]. Due to the complexity of the therapy it is recommended that the therapy is performed in a specialist hospital only in order to be able to immediately react to side effects. How long a patient should be hospitalized, however, must be decided according to the exact CAR-T therapy, fitness, clinical stability and psychosocial support of the patient. Improved CAR-T cells that are safer for patients are under development.

\section{Discussion}

Due to the resounding successes of the targeted modulation of the immune system by adoptive immunotherapies like monoclonal antibodies and checkpoint inhibitors, there was great hope to develop such a successful therapy for leukemia as well. So far, some leukemia-specific monoclonal antibodies have become available, however, development of effective checkpoint antibodies to therapy-refractory/relapsed leukemia has not yet been achieved. Immunotherapies based on CAR-T 
Citation: Susanne Reuther. Genetically modified adoptive immunotherapy-A new therapeutic option to cure relapsed and/or refractory leukemia in children and adults? J Med Oncl Ther 2018;3(2):36-45.

cells go one step further by engineering the $\mathrm{T}$ cell receptor itself to enhance the response of the immune system against a specific tumor/leukemia antigen. The clinical benefit of immunotherapies appears to depend on whether tumor escape mechanisms can be overcome. Tumor escape reactions are mainly caused by the selective pressure of an immune response, which means that the more advanced a malignancy is, the more escape mechanisms have already been formed.

Adoptive immunotherapies are more specific in targeting cancer cells compared to traditional methods like toxic chemotherapy and radiation. With the development of CAR-T cell therapy, immunotherapy has gained another possibility to eliminate cancer cells. CAR-T cell therapy is the most promising approach which has shown remarkable ability to eliminate various kinds of cancers with successful response rates and durable complete remission. However, all these therapies can also trigger mild to very severe side effects and may severely limit the quality of life. Furthermore, they can only be used for subsets of patients, of which not every patient responds to therapy. Further investigation is needed to optimize tumor specificity and minimize side effects.

\section{Conclusion}

The field of immunotherapy for leukemia and other cancers is rapidly expanding. While the new treatment options can trigger very successful effects, they also come with downsides and might not be safe for each patient.

Guidelines and expert recommendations highlight that CAR-T immunotherapy is promising in most high-risk cases of leukemia, but for optimal results accurate patient selection, best preparation, monitoring and follow-up are required.

Nonetheless, it is likely that CAR-T cells will also be approved for treatment of other types of cancer, including solid tumors, if positive results are obtained in clinical trials. This would allow the application of this completely new adoptive immunotherapy in a variety of cancer types.

\section{References}

1. Vardiman JW, Harris NL, Brunning RD. The World Health Organization (WHO) classification of the myeloid neoplasms. Blood. 2002;100:2292-302.

2. Vardiman JW, Thiele J, Arber DA, et al. The 2008 revision of the World Health Organization (WHO) classification of myeloid neoplasms and acute leukemia: rationale and important changes. Blood. 2009;114:937-51.

3. Hehn. Frequency of leukemia in Germany. Kompetenznetz Leukämie. 2014.

4. Kaatsch P, Spix C, Grabow D, et al. Jahresbericht annual report 2017 (1980-2016). Deutsches Kinderkrebsregister DKKR. German Childhood Cancer Registry GCCR. 2017.

5. Noone AM, Howlader N, Krapcho M, et al. SEER cancer statistics review, 1975-2015. National Cancer Institute. Bethesda. 2015.
6. Kaatsch P, Spix C, Grabow D, et al. Jahresbericht annual report 2016 (1980-2016). Deutsches Kinderkrebsregister DKKR. German Childhood Cancer Registry GCCR. 2016.

7. Jackson N, Menon BS, Zarina W, et al. Why is acute leukemia more common in males? A possible sexdetermined risk linked to the ABO blood group genes. Ann Hematol. 1999;233-36.

8. Smith MA, Altekruse SF, Adamson PC, et al. Declining childhood and adolescent cancer mortality. Cancer. 2014;120: 2497-506.

9. PDQ Pediatric Treatment Editorial Board, (NIH) BNCI. Childhood acute lymphoblastic leukemia treatment (PDQ $\left.{ }^{\circledR}\right)$ : health professional version. PDQ Cancer Information Summaries. 2018.

10. Rubnitz JE. How I treat pediatric acute myeloid leukemia. Blood. 2012;119:5980-88.

11. Colby-Graham MF, Chordas C. The childhood leukemias. J Pediatr Nurs. 2003;18:87-95.

12. Creutzig U, Zimmermann M, Hannemann J, et al. Quality assurance in the competence network pediatric oncology and hematology. Klin Pediatrics. 2003; 215:338-40.

13. Niewerth D, Creutzig U, Bierings MB, et al. A review on allogeneic stem cell transplantation for newly diagnosed pediatric acute myeloid leukemia. Blood. 2010;116:2205-14.

14. Nemecek ER, Guthrie KA, Sorror ML, et al. Conditioning with treosulfan and fludarabine followed by allogeneic hematopoietic cell transplantation for high-risk hematologic malignancies. Biol Blood Marrow Transplant. 2011;17:341-50.

15. Stam RW, den Boer ML, Pieters R. Towards targeted therapy for infant acute lymphoblastic leukaemia. Br J Haematol. 2006;132:539-51.

16. Bachas C, Schuurhuis GJ, Hollink IH, et al. Highfrequency type I/II mutational shifts between diagnosis and relapse are associated with outcome in pediatric AML: Implications for personalized medicine. Blood. 2010;116:2752-8.

17. Aversa F, Martelli MF. Transplantation of haploidentically mismatched stem cells for the treatment of malignant diseases. Springer Semin Immunopathol. 2004;26:155-68.

18. Müller S, Friedrich W. Stem Cell Transplantation for Treatment of Primary Immunodeficiency Disorders. Iran J Allergy Asthma Immunol. 2005; 4:1-8.

19. Miano M, Labopin M, Hartmann O, et al. Haematopoietic stem cell transplantation trends in children over the last three decades: a survey by the paediatric diseases working party of the European Group for Blood and Marrow Transplantation. Bone Marrow Transplant. 2007;39:89-99

20. Gluckman E, Rocha V. Cord blood transplantation for children with acute leukaemia: A Eurocord registry analysis. Blood Cells Mol Dis. 2004;33:271-3.

21. Ho VT, Soiffer RJ. The history and future of T-cell depletion as graft-versus-host disease prophylaxis for allogeneic hematopoietic stem cell transplanta. Blood. 2001;98:3192-204. 
22. Kolb HJ, Mittermüller J, Clemm C, et al. Donor leukocyte transfusions for treatment of recurrent chronic myelogenous leukemia in marrow transplant patients. Blood. 1990;76:2462-5.

23. Collins SP, Shpilberg O, D'robyski WR, et al. Donor leukocyte infusions in 140 patients with relapsed malignancy after allogeneic bone marrow transplantation. J Clin Oncol. 1997;15:433-44.

24. Porter DL, Collins RH, Shpilberg O, et al. Long-term follow-up of patients who achieved complete remission after donor leukocyte infusions. Biol Blood Marrow Transplant. 1999:253-61.

25. Carlens S, Remberger M, Aschan J, et al. The role of disease stage in the response to donor lymphocyte infusions as treatment for leukemic relapse. Biol Blood Marrow Transplant. 2001;7:31-38

26. Kolb HJ, Schattenberg A, Goldman JM, et al. Graftversus-leukemia effect of donor lymphocyte transfusionsin marrow grafted patients. Blood. 1995;86:2041-50.

27. Riddell S, Murata M, Bryant $S$ et al. Minor histocompatibility antigens targets of graft versus leukemia responses. Int J Hematol. 2002;76:155-61.

28. Szydlo R, Goldman JM, Klein JP, et al. Results of allogeneic bone marrow transplants for leukemia using donors other than hla-identical siblings. JClin Oncol. 1997; 15: 1767-77.

29. Vago L, Perna SK, Zanussi M, et al. Loss of mismatched HLA in Leukemia after stem-cell transplantation. N Engl J Med. 2009;361:478-88.

30. Kolb HJ, Schmid C, Barrett AJ et al. Graft-versusleukemia reactions in allogeneic chimeras. Blood. 2004;103:767-76.

31. Schuster FR, Meisel R, Fuhrer M, et al. Anti-leukaemic activity of a novel haploidentical-transplantation approach employing unmanipulated bone marrow followed by CD6depleted peripheral blood stem cells in children with refractory/relapsed acute leukaemia. $\mathrm{Br} \mathrm{J}$ Haematol. 2013;162:802-7.

32. Shiobara S, Nakao S, Ueda M, et al. Donor leukocyte infusion for Japanese patients with relapsed leukemia after allogeneic bone marrow transplantation: lower incidence of acute graft-versus-host disease and improved outcome. Bone Marrow Transplantation. 2000;769-74

33. Shiobara S, Nakao S, Ueda M, et al. Donor leukocyte infusion for Japanese patients with relapsed leukemia after allogeneic bone marrow transplantation: Indications and dose escalation. Therapeutic Apheresis. 2001;5:40-5.

34. Ruggeri L, Capanni M, Urbani E et al. Effectiveness of donor natural killer cell alloreactivity in mismatched hematopoietic transplants. Science. 2002;295.

35. Alatrash G, Jeffrey JM. Immunotherapy of AML. Cancer Treat Res. 2010;237-55.

36. Li Y, Sun R. Tumor immunotherapy: New aspects of natural killer cells. Chin J Cancer Res. 2018;30:173-96.

37. Bekisz J, Baron S, Balinsky C, et al. Antiproliferative properties of Type I and Type II interferon. pharmaceuticals (Basel) 2010;3:994-1015.
38. Talpaz M, Mercer J, Hehlmann R. The interferon-alpha revival in CML. Ann Hematol. 2015;2:195-207.

39. Talpaz M, Shah NP, Kantarjian H, et al. Dasatinib in imatinib-resistant Philadelphia chromosome-positive leukemias. N Engl J Med. 2006;354:2531-4.

40. Mubarak AAS, Kakil IR, Awidi A, et al. normal outcome of pregnancy in chronic myeloid leukemia treated with interferon-In 1st Trimester: Report of 3 cases and review of the literature. Am J Hematol. 2002;69:115-8.

41. Okanoue T, Sakamoto S, Itoh Y, et al. Side effects of highdose interferon therapy for chronic hepatitis C. J Hematol. 1996;25:283-91.

42. Conlon KC, Miljkovic MD, Waldmann TA. Cytokines in the treatment of cancer. J Interferon Cytokine Res. 2018.

43. National Cancer Institute (NIH). Adult acute myeloid leukemia treatment. National Cancer Institute at the National Institute of Health. 2013.

44. Cheson B, Leonard JP. Monoclonal Antibody therapy for B-cell non-hodgkins lymphoma. $N$ Engl J Med. 2008;359:613-26.

45. Scott AM, Wolchok JD, Old LJ. Antibody therapy of cancer. Nat Rev Cancer. 2012;12:278-87.

46. Hoelzer D. Novel antibody-based therapies for acute lymphoblastic leukemia. Current management issues in acute lymphocytic leukemia. Blood. 2011; 243-9.

47. Ferreira AF, de Oliveira GL, Tognon R, et al. Apoptosisrelated gene expression profile in chronic myeloid leukemia patients after imatinib mesylate and dasatinib therapy. Acta Haematol. 2015;133:354-64.

48. Zitvogel L, Rusakiewicz S, Routy B, et al. Immunological off-target effects of imatinib. Nat Rev Clin Oncol, 2016;13:431-46.

49. Ongoren S, Eskazan AE, Suzan V, et al. Third-line treatment with second-generation tyrosine kinase inhibitors (dasatinib or nilotinib) in patients with chronic myeloid leukemia after two prior TKIs: Real-life data on a single center experience along with the review of the literature. Hematology. 2018;23:212-20.

50. Keating GM. Dasatinib: A review in chronic myeloid leukaemia and $\mathrm{Ph}+$ acute lymphoblastic leukaemia. Drugs. 2017;77:85-96.

51. Baccarani M, Castagnetti F, Gugliotta G, et al. A review of the European LeukemiaNet recommendations for the management of CML. Ann Hematol. 2015; 94:141-7.

52. Kong JH, Khoury HJ, Kim AS, et al. The safety of Bosutinib for the treatment of chronic myeloid leukemia. Expert Opin Drug Saf. 2017;16:1203-9.

53. Muller MC, Cervantes F, Hjorth-Hansen $\mathrm{H}$, et al. Ponatinib in chronic myeloid leukemia (CML): Consensus on patient treatment and management from a European expert panel. Crit Rev Oncol Hematol. 2017;120:52-9.

54. De Weerdt I, Koopmans SM, Kater AP, et al. Incidence and management of toxicity associated with ibrutinib and idelalisib: A practical approach. Haematologica. 2017;102:1629-39. 
Citation: Susanne Reuther. Genetically modified adoptive immunotherapy-A new therapeutic option to cure relapsed and/or refractory leukemia in children and adults? J Med Oncl Ther 2018;3(2):36-45.

55. Barrientos JC. Idelalisib for the treatment of indolent nonHodgkin lymphoma: A review of its clinical potential. Onco Targets Ther. 2016;9:2945-53.

56. Zahavi D, AlDeghaither D, O’Connell A, et al. Enhancing antibody-dependent Cell-mediated cytotoxicity (ADCC)A strategy for improving antibody-based immunotherapy. Antibody Therapeutics. 2018;1:7-12.

57. Schnitzler M, Hasskarl J, Egger M, et al. Successful treatment of severe acute intestinal graft-versus-host resistant to systemic and topical steroids with alemtuzumab. Biol Blood Marrow Transplant. 2009;15:910-18.

58. Tanaka M, Kano Y, Akutsu M, et al. The cytotoxic effects of gemtuzumab ozogamicin (mylotarg) in combination with conventional antileukemic agents by isobologram analysis in vitro. Anticancer Res. 2009:4589-96.

59. O'Hear C, Heiber JF, Schubert I, et al. Anti-CD33 chimeric antigen receptor targeting of acute myeloid leukemia. Haematologica. 2015;100:336-44.

60. Kantarjian HM, DeAngelo DJ, Stelljes $M$, et al. Inotuzumab ozogamicin versus standard therapy for acute lymphoblastic leukemia. N Engl J Med. 2016;375:740-53.

61. Dang NH, Ogura M, Castaigne S, et al. Randomized, phase 3 trial of inotuzumab ozogamicin plus rituximab versus chemotherapy plus rituximab for relapsed/ refractory aggressive B-cell non-Hodgkin lymphoma. Br J Haematol. 2018;182:583-6.

62. Tobinai K, Klein C, Oya N, et al. A Review of Obinutuzumab (GA101), a novel type II Anti-CD20 monoclonal antibody, for the treatment of patients with BCell malignancies. Adv Ther. 2017;34:324-56.

63. Goede V, Fischer K, Busch R, et al. Obinutuzumab plus chlorambucil in patients with CLL and coexisting conditions. N Engl J Med. 2014;370:1101-10.

64. Bologna L, Gotti E, Da Roit F, et al. Ofatumumab is more efficient than rituximab in lysing $\mathrm{B}$ chronic lymphocytic leukemia cells in whole blood and in combination with chemotherapy. J Immunol. 2013;190:231-9.

65. Shimada K, Tomita A, Saito S, et al. Efficacy of ofatumumab against rituximab-resistant B-CLL/SLL cells with low CD20 protein expression. $\mathrm{Br} \mathrm{J}$ Haematol. $2014 ; 166$

66. Glaxo SK. Drug Commission of the German medical profession. Drug Safety Mail 2013-66 -Rote-Hand-Brief on Arzerra ${ }^{\circledR}$ (Ofatumumab): Hepatitis B virus (HBV) screening before treatment. 2013.

67. Forstpointner R, Unterhalt $M$, Dreyling $M$, et al. Maintenance therapy with rituximab leads to a significant prolongation of response duration after salvage therapy with a combination of rituximab, fludarabine, cyclophosphamide, and mitoxantrone (R-FCM) in patients with recurring and refractory follicular and mantle cell lymphomas: Results of a prospective randomized study of the German Low Grade Lymphoma Study Group (GLSG). Blood. 2006;108:4003-8.

68. Berinstein NL, Grillo-Lopez AJ, White CA, et al. Association of serum Rituximab (IDEC-C2B8) concentration and anti-tumor response in the treatment of recurrent low-grade or follicular non-Hodgkin's lymphoma. Annals Oncol. 1998;9:995-1001.

69. Maloney DG, Grillo-Lopez AJ, White CA, et al. IDECC2B8 (Rituximab) Anti-CD20 monoclonal antibody therapy in patients with relapsed low-grade non-hodgkin's lymphoma. Blood. 1997;90:2188-95.

70. Byrd KP, Vontela NR, McCullar B, et al. Multi-database description of primary splenic diffuse large b-cell lymphoma. Anticancer Res. 2017; 37:6839-43.

71. Salles G, Barrett M, Foa R, et al. Rituximab in B-Cell hematologic malignancies: A review of 20 years of clinical experience. Adv Ther. 2017; 34: 2232-73.

72. Dartigeas C, Van Den Neste E, Léger J, et al. Rituximab maintenance versus observation following abbreviated induction with chemoimmunotherapy in elderly patients with previously untreated chronic lymphocytic leukaemia (CLL 2007 SA): An open-label, randomised phase 3 study. Lancet Haematol. 2017;1:1-13.

73. Topp MS, Kufer P, Gökbuget N, et al. Targeted therapy with the t-cell-engaging antibody blinatumomab of chemotherapy-refractory minimal residual disease in blineage acute lymphoblastic leukemia patients results in high response rate and prolonged leukemia-free survival. J Clinical Oncol. 2011;29:2493-8.

74. Bargou R, Leo E, Zugmaier G, et al. Tumor regression in cancer patients by very low doses of a $\mathrm{T}$ cell-engaging antibody. Science. 2008;321:974-7.

75. Postow MA, Callahan MK, Wolchok JD. Immune checkpoint blockade in cancer therapy. J Clin Oncol. 2015;33:1974-82.

76. Littman DR. Releasing the brakes on cancer immunotherapy. Cell. 2015;162:1186-90.

77. Meiliana A, Dewi NM, Wijaya A. Cancer immunotherapy: A review. Cancer Immunother. 2016;8:1-20.

78. Caroline RC, Thomas L, Bondarenko I, et al. Ipilimumab plus dacarbazine for previously untreated metastatic melanoma. N Engl J Med. 2011; 364:2517-26.

79. Hodi FS, O'Day SJ, McDermott DF, et al. Improved survival with ipilimumab in patients with metastatic melanoma. N Engl J Med. 2010;363:711-23.

80. Ribas A, Hodi FS, Kefford R, et al. Efficacy and safety of the anti-PD-1 monoclonal antibody MK-3475 in 411 patients (pts) with melanoma (MEL). J Clin Oncol. 2014:32.

81. Robert C, Long GV, Brady B, et al. Nivolumab in previously untreated melanoma without BRAF mutation. N Engl J Med. 2015; 372:320-30.

82. Boddu P, Kantarjian H, Garcia-Manero G, et al. The emerging role of immune checkpoint based approaches in AML and MDS. Leuk Lymphoma. 2018;59:790-802.

83. Vairy S, Garcia JL, Teira P, et al. CTL019 (tisagenlecleucel): CAR-T therapy for relapsed and refractory B-cell acute lymphoblastic leukemia. Drug Des Devel Ther. 2018;12:3885-98.

84. Pehlivan KC, Duncan BB, Lee DW. CAR-T Cell Therapy for acute lymphoblastic leukemia: Transforming the 
treatment of relapsed and refractory disease. Curr Hematol Malig R. 2018;13:396-406

85. Ramos CA, Savoldo B, Dotti G. CD19-CAR Trials. Cancer Res. 2014; 20: 112-8.

86. Maude SL, Frey N, Shaw PA, et al. Chimeric antigen receptor $\mathrm{T}$ cells for sustained remissions in leukemia. $\mathrm{N}$ Engl J Med. 2014; 371: 1507-17.

87. Davila ML, Riviere I, Wang X, et al. Efficacy and toxicity management of 19-28z CAR T cell therapy in B cell acute lymphoblastic leukemia. Sci Transl Med. 2014; 6: 224-5.

88. Thomas X, Paubelle E. Tisagenlecleucel-T for the treatment of acute lymphocytic leukemia. Expert Opin Biol Ther. 2018;18:1095-106.

89. Fry TJ, Shah NN, Orentas RJ, et al. CD22-targeted CAR T cells induce remission in B-ALL that is naive or resistant to CD19-targeted CAR immunotherapy. Nat Med. 2018;24:20-28.
90. Buchholz CJ, Hartmann J, Schüßler-Lenz M, et al. CART- cell therapy-prospects and risks. Ger Med J.

91. Brudno JN, Kochenderfer JN. Toxicities of chimeric antigen receptor $\mathrm{T}$ cells: Recognition and management. Blood. 2016;127:3321-30.

92. Mahadeo KM, Khazal SJ, Abdel-Azim H, et al. Management guidelines for paediatric patients receiving chimeric antigen receptor $\mathrm{T}$ cell therapy. Nat Rev Clin Oncol. 2019;16:45-63.

\section{*Correspondence to:}

Susanne Reuther

Ludwig-Maximillian University Munich, Medical Faculty

University Hospital of Munich

E-mail: susanne.reuther@campus.lmu.de 